\title{
LA OPTIMIZACIÓN DEL APRENDIZAJE EN LA UNIVERSIDAD A TRAVÉS DE SU ARQUITECTURA: PLANIFICACIÓN, ESCALA HUMANA Y COMUNIDAD VIVENCIAL
}

\section{The optimization of learning in the university through its architecture: planning, buman scale and living community}

Pablo Campos Calvo-Sotelo

Universidad San Pablo CEU

Correo-e: utoplan@telefonica.net

Recepción: 5 de mayo de 2017

Envío a informantes: 6 de junio de 2017

Aceptación definitiva: 30 de noviembre de 2017

Resumen: La formación humana, canalizada a través de procesos de enseñanza-aprendizaje, es una experiencia necesariamente espacial; esta convicción constituye el fundamento teórico primordial sobre el que se sustenta el presente artículo. En aras de optimizar la motivación de los estudiantes hacia su formación, resulta trascendental combinar tres de los principios del paradigma del Campus Didáctico: planificación espacial, escala humana y comunidades vivenciales de aprendizaje e investigación. Mediante el proceso de planificación, las universidades pueden dibujar una evolución a largo plazo de sus lugares, prestando especial atención a aquellos caracterizados por su escala humana. Con tan sólida estrategia, los alumnos incrementarán su sentimiento de pertenencia para con la institución, construyendo con facilidad verdaderas comunidades de aprendizaje e investigación. Con todo ello, el propósito del presente texto es incidir en la necesidad de concebir de cara al futuro formatos arquitectónicos en clave de Campus Didáctico, que respondan desde la innovación y la sensibilidad a la situación y las tendencias contemporáneas en la educación superior, enriqueciendo tanto la motivación de cara a formación que han de protagonizar sus usuarios, como su trascendental proyección social.

Palabras clave: arquitectura; educación; universidad; innovación; campus didáctico; planificación espacial. 
АвsтRAст: Higher education, channelized through teaching \& learning processes, is a necessary spatial experience; this conviction constitutes the essential theoretical foundation which supports the present article. With the intention of optimizing motivation of students towards their formation, it becomes transcendental to combine three of the principles of the Educational Campus paradigm: spatial planning, human scale and existential communities of learning \& research. Through the planning process, universities can draw long term evolution of their places, paying special attention to those characterized by their human scale. With such a sound strategy, students will increase their feeling of belonging towards the Institution, building up easily true communities of learning \& research. With all these topics in mind, the purpose of the present text is to remark for the future the need of conceiving higher education spaces inspired on the concept of Educational Campus, which respond from innovation and sensitiveness to the contemporary situation and trends, enriching both the motivation of its users towards formation as well as its transcendental social projection.

KeY words: Architecture; Education; University; innovation; educational campus; spatial planning.

\section{Introducción: el rol de la arquitectura en la optimización de la educación superior}

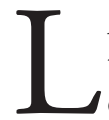

A COYUNTURA UNIVERSITARIA ACTUAL a escala internacional recomienda efectuar una labor de investigación, centrada tanto en los fundamentos teóricos como en sus aplicaciones proyectuales, que contribuya a optimizar la calidad de cuanto acontece dentro de los recintos docentes. El objetivo de estas indagaciones ha de ser justificar y reivindicar la necesidad de generar espacios físicos de calidad como aval para la paralela calidad de los procesos formativos de la educación superior. Y teniendo bien presente algo sustancial: que el destino de tales esfuerzos de investigación y praxis constructiva es reforzar un aspecto determinante en la Universidad, cual es la motivación del estudiante. Una motivación que le servirá para que pueda desarrollar un aprendizaje satisfactorio, una de cuyas claves es la cristalización de ambientes urbanístico-arquitectónicos y humanos (comunidades), que incrementen su sentimiento de pertenencia para con la institución que albergue tales procesos formativos. Existen, en suma, tres estrategias que pueden ser puestas al servicio de las universidades para alcanzar estos fines: planificación, refuerzo de la escala humana y cristalización de verdaderas comunidades de aprendizaje. Sobre la conveniencia de ser valoradas en la coyuntura actual y sobre el modo en que están concatenadas versa buena parte del presente texto.

La educación es un hecho que debe responder a un conjunto de cualidades. En primer término, ha de valerse de una afectividad en la relación profesor/alumno, de modo que exista una cercanía mutua que, sin menoscabar el rigor y la exigencia, conduzca el proceso formativo por cauces de motivación y sensibilidad. En un segundo plano de análisis, no se pueden desaprovechar las ingentes oportunidades derivadas del aprendizaje en colectividad, construyéndose verdaderas comunidades docentes y de investigación que se alejen del campus virtual; los riesgos inherentes a semejante tendencia ya han sido exhaustivamente señalados por destacados expertos, como la arquitecta argentina Marina Waisman, quien dejaba clara su postura sobre los riesgos de la disolución de la materialidad: 
LA OPTIMIZACIÓN DEL APRENDIZAJE EN LA UNIVERSIDAD A TRAVÉS DE SU ARQUITECTURA: PLANIFICACIÓN, ESCALA HUMANA Y COMUNIDAD VIVENCIAL PABLO CAMPOS CALVO-SOTELO

$\mathrm{Al}$ abolir la materialidad, sustituida por la mera imagen o, más aún, por el simulacro, se anula la relación tiempo/espacio, se suprime el espacio. [...] Así, tiempo y espacio, las coordenadas que permiten al individuo representar su situación con relación a la totalidad, se disuelven, dejándolo perdido en el vacío. Y esta ha sido llamada, precisamente, la era del vacío (Lipovetsky) (Waisman, 1995: 68).

En consecuencia, a esta dimensión presencial del colectivo que se entrega al aprendizaje va a dedicarse buena parte del presente texto, ya que dicha presencialidad atesora valiosas virtudes que ayudan a que la Universidad cumpla su verdadera misión, cual es la construcción de profesionales en clave de personas éticamente comprometidas con su sociedad. Puesto que se concluye de lo anterior que el contacto humano es imprescindible para alcanzar las cualidades descritas en la educación superior, no puede soslayarse la presencia de un marco construido que acoja todo ello. Así, la educación ha de asumirse necesariamente como un hecho de carácter presencial y espacial, que necesita de los marcos edificados para albergar el roce interpersonal. Un rector español afirmaba hace pocos años:

El Lugar de la Sabiduría nos evoca el espacio donde transcurre nuestra vida académica, recordándonos que la educación tiene una dimensión espacial; que la formación de las personas, como misión de la Universidad, precisa de un entorno construido que ha de poner de manifiesto sus virtudes socializadoras e integradoras; que tiene que definir la escala de valores, desarrollar los estímulos psicológicos y permitir, en definitiva, a los grupos de personas proyectar y expresar sus aspiraciones tanto personales como colectivas (Abril, 2010: 9).

Ahora bien, ¿es este el único rol que cabe exigir a la arquitectura? La negativa respuesta a este interrogante ha de acompañarse con el argumento de que, trascendiendo su mero papel como contenedor material donde se albergan las actividades inherentes a la educación superior, su dimensión espacial es un componente capaz de desencadenar por sí mismo procesos cognitivos innovadores. Y de transmitir sus propios valores como obra artística. Esto último implica un salto cualitativo, ya que se incorpora una primordial faceta didáctica que -referida a todo tipo de funciones arquitectónicas- ha sido ensalzada por autores como Franco Purini, en su trabajo La arquitectura didáctica (Purini, 1984). Para que las universidades acometan con un bagaje argumental suficiente sus dinámicas de progreso hacia la excelencia, se ha generado una herramienta conceptual y operativa, el Campus Didáctico, útil para la descrita tarea de acometer procesos globales hacia la optimización de sus estructuras.

\section{I.I. El Campus Didáctico como estímulo proyectual}

Fruto de investigaciones recientes, se ha formulado el Campus Didáctico, como fundamento filosófico, pero de vocación pragmática, cuya misión es la de contribuir a impulsar procesos de transformación cualitativa en recintos universitarios. Fue enunciado inicialmente en 2005, con motivo del diseño del nuevo recinto de Villamayor (Universidad de Salamanca). A partir de su génesis, se divulgó internacionalmente, en diversas publicaciones: revista de la OCDE Programme on Educational Building-Exchange (Campos, 2005); revista La Cuestión universitaria (Campos, 2009); revista 
Aula-Revista de Pedagogía de la Universidad de Salamanca (Campos, 20Ioa); libro España. Campus de Excelencia Internacional (Campos, 201ob); libro Composición Arquitectónica. Fundamentos teóricos y aplicaciones en los espacios para la Educación (Campos, 20I2); revista CIAN-Universidad Carlos III de Madrid (Campos, 20I4); publicación Arquitectonics (Campos, 20I5); revista Relapae (Campos, 2017); o su inclusión dentro de las bases del Programa Campus de Excelencia Internacional, desarrollado en 2009, 2010 y 2011 por el Ministerio de Educación de España.

Posteriormente, tanto mediante investigaciones como a través de su empleo como estímulo en proyectos universitarios, se perfiló su renovada estructura orgánica, resultando una versión actualizada de sus principios, que constituyeron el corpus de una reciente tesis doctoral (Campos, 20I7); se enumeran seguidamente:

I. Fundamentación en la Utopía.

2. Génesis y evolución de una acción planificadora integral.

3. Cristalización de una comunidad vivencial de aprendizaje e investigación.

4. Configuración global unitaria, compatible con la diversidad individual.

5. Consecución de una dimensión estética de orden urbanistico-arquitectónico.

6. Incorporación de ámbitos de escala bumana.

7. Presencia activa y múltiple de la Naturaleza.

8. Integración y promoción del Arte.

9. Proyección de rasgos simbólicos.

Io. Apertura al entorno y accesibilidad al aprendizaje.

II. Armonización formal y conceptual con el lugar preexistente.

I2. Aplicación de criterios y estrategias relativas a la sostenibilidad.

13. Consideración de la memoria tipológica en clave de interpretación.

I4. Recualificación patrimonial y funcional.

15. Impulso del desarrollo e innovación inducidos.

16. Compromiso con el vanguardismo urbanístico-arquitectónico.

17. Optimización de las sinergias entre Universidad y Ciudad.

18. Activación de espacios para la incorporación de modalidades innovadoras de Enseñanza/Aprendizaje.

El telón de fondo de toda esta relación de cualidades que debe atesorar un recinto universitario para alcanzar su pleno rol como Campus Didáctico está dibujado por la trascendencia que posee la formación integral. Efectivamente, y como se ha anticipado líneas atrás, al efectuar una aproximación a dicha formación bajo una impronta de innovación, se colige inmediatamente que ha de efectuarse dentro de la arquitectura, asumida como componente ineludible de todo proceso educativo.

El Campus Didáctico quiere afianzarse como un recurso que impulse procesos de creatividad aplicada a los espacios físicos de la Universidad. Tal pretensión no se explica tanto porque cada uno de los principios que lo componen sean intrínsecamente innovadores en sí mismos, sino porque lo verdaderamente creativo radica en la comprensión conjunta de todos esos principios a la vez, integrados en una estructura de pensamiento y acción unitaria. Pues bien, y partiendo de estas consideraciones básicas, puede comentarse que la dimensión didáctica está intrínsecamente ligada a la arquitectura, en tanto que Arte que posee entre sus inconmensurables virtudes la de valerse de su propia historia como hecho formativo. Como expone Purini: «La 
LA OPTIMIZACIÓN DEL APRENDIZAJE EN LA UNIVERSIDAD A TRAVÉS DE SU ARQUITECTURA: PLANIFICACIÓN, ESCALA HUMANA Y COMUNIDAD VIVENCIAL PABLO CAMPOS CALVO-SOTELO

dimensión didáctica es el lugar conceptual en que la arquitectura, instrumento de la vida cotidiana y sostén de la vida heroica, reencuentra continuamente su propio origen» (Purini, I984: III). Otro modo de justificar la misión formativa que deben poseer los espacios construidos per se, expresado en clave pedagógica, pasa por considerar a la arquitectura como una «forma tácita de enseñanza», expresión propuesta por el pedagogo italiano Mauro Laeng (Laeng, 1977). Deben existir sinergias entre docencia y arquitectura, explicables en clave de «trama» de interacción, dentro de la cual las edificaciones debieran desempeñar un papel pedagógico fehaciente: la arquitectura de la Universidad ha de educar per se, actuando como «libro de texto tridimensional» (Kong, Yaacob y Ariffin, 2015). Así asumido, el medio arquitectónico «se hace lugar y educa» (Romañá, 2004: 207).

En lo que atañe a sus ámbitos de aplicación, el Campus Didáctico podrá hacerse presente en la tétrada convencional de escalas universitarias: la relación Universidad/ Ciudad, el recinto, el edificio y el aula. En tal sentido, debe efectuarse una matización relevante, que afecta a la interconexión entre ellas, defendiendo que la arquitectura y el urbanismo conforman una entidad sin solución de continuidad. Ambas realidades construidas tienen un mismo cometido: ordenar el espacio. Las divergencias, si las hubiere en lo sustancial, se remiten a una cuestión meramente escalar, de corte cuantitativo, que no debe distraer de su esencial denominador común. Como acertadamente señalaba el arquitecto Julio Cano Lasso: «Empecemos por decir que urbanismo y arquitectura son una misma cosa» (Cano, 1993: 34).

En función de todo lo expuesto, se procede seguidamente a establecer un discurso transversal que, apoyándose en tres de los principios del Campus Didáctico, culmine en la demostración de la conveniencia de adoptar una estrategia específica de ideación: establecer las pautas para que las universidades puedan potenciar la motivación del alumnado de cara al aprendizaje, en forma de comunidades dentro de las cuales la arquitectura ayude a incrementar el trascendental sentimiento de pertenencia.

\section{La planificación urbanístico-arquitectónica como requisito}

Planificar es una cualidad imprescindible para que la Universidad progrese y se proyecte al futuro dotada de la suficiente capacidad de modificarse según se vean alteradas las condiciones de su entorno. Se trata de una acción imprescindible para avalar itinerarios coherentes en los recintos universitarios. Así entendida, la elaboración de estrategias de planificación integral de la Universidad define tanto la creación ex novo de sus sedes físicas, como un desarrollo de las mismas dotado de un marcado índice de libertad y flexibilidad espacio/temporal. Se ha ido consolidando históricamente como un requisito capaz de impulsar la vitalidad de los recintos docentes sin incurrir en contradicciones. Un repaso a la historia de las instituciones dedicadas a la educación superior testimonia que su avance ha estado asociado a una actitud racional de ideación y proyección de sus estructuras espaciales. Entre los extraordinarios ejemplos que ha dejado la memoria de la planificación, cabe recordar el caso de la Civitas Dei de Alcalá, donde en 1499 grabó su magistral huella el Cardenal Cisneros. Una magnífica ilustración de la planificación contemporánea es la Universidad Libre de Berlín. Fundada en 1948, esta institución efectuó en 1963 una de las planificaciones más trascendentes del siglo xx, erigiéndose en paradigma de la 
trama como mecanismo de composición arquitectónica, bajo el diseño de Candilis, Josic y Woods.

La importancia de esta cuestión ha llevado incluso a algunos autores a defender su perfil artístico. En su trabajo The campus as a work of Art, Thomas Gaines argumentaba que la planificación de un campus constituye un acto de creación artística, asignándole incluso un nivel como dimensión: «A diferencia de la dimensión bidimensional de la pintura, la tridimensionalidad del arte escultórico, y la arquitectura, donde la cuarta dimensión es la función, el campus posee una quinta dimensión: la planificación» (Gaines, I991: ix).

La planificación emerge en el escenario universitario como una actitud crítica cuando se haya de proyectar la incorporación de ámbitos de perfil acogedor e intimista, cuestión que se considera en el presente texto como factor decisivo para alcanzar la cristalización de comunidades de aprendizaje dotadas de un sólido componente humano. Tanto el nacimiento como la posterior progresión de un recinto universitario han seguido históricamente un conjunto de tipologías que, aun no siendo elemento central del presente artículo, merecen ser glosadas. Los trazados iniciales suelen recurrir a estos modelos compositivos: reticular, radioconcéntrico, lineal, nuclear, orgánico, entre otros posibles y sus combinaciones. Por otro lado, la evolución presenta las siguientes situaciones prototípicas: polarización, transformación, extensión, densificación, rehabilitación, adaptación, centrifugación y collage (Campos, 2012: 89).

En cuanto a su aplicación, planificar afecta a las mencionadas cuatro esferas de la Universidad (ciudad, recinto, edificio y aula). Abarcándolas globalmente, se disuelven los límites entre ellas, y se ordena armónicamente la sucesión de ambientes y sus eventuales alteraciones en el tiempo. Este conjunto multiescalar abarca la dimensión intimista, como se ha apuntado anteriormente, lo que redundará en que se incremente el sentimiento de pertenencia del alumno y la consiguiente motivación.

En sí mismo, el hecho de planificar es susceptible de entenderse bajo diferentes ópticas. El doctor Castrejón la interpretaba así: «Con la planificación se pretende introducir orden y dirección en el proceso de cambio» (Castrejón, I990: 173). La incorporación del principio de orden entronca directamente con otra cualidad exigible al cuerpo físico de la Universidad, cual es el de la armonía espacial; la idea de dirección remite al dinamismo evolutivo.

La elaboración de planes para un recinto universitario ha de realizarse bajo una premisa de flexibilidad. Esta faceta incide directamente en la conformación de ámbitos de escala humana, susceptibles de ir amoldándose a las variaciones del colectivo estudiantil y -por tanto- reforzando la identificación del mismo con los contextos construidos donde aprende. Este concepto de flexibilidad, inexorablemente ligado a la variabilidad funcional de la educación superior, debe trasladarse necesariamente a su corpus edificado:

La aceptación de una integración de las estructuras universitarias en la multiforme y compleja experiencia de un mundo en rápida evolución, el reconocimiento de una exigencia de flexibilidad y adaptabilidad a las condiciones diversas, cambiantes en el tiempo y en el espacio... (Rebecchini, 198r: 6).

El hecho de que la planificación (sobre todo en Europa) no ha sido tratada en las últimas décadas con la energía deseable queda patente al releer aportaciones de 
LA OPTIMIZACIÓN DEL APRENDIZAJE EN LA UNIVERSIDAD A TRAVÉS DE SU ARQUITECTURA: PLANIFICACIÓN, ESCALA HUMANA Y COMUNIDAD VIVENCIAL PABLO CAMPOS CALVO-SOTELO

hace casi 50 años, en las cuales se proponían estrategias que todavía hoy no se activan debidamente. Como muestra, el arquitecto italiano Giancarlo de Carlo ya defendía la flexibilidad hace décadas, introduciendo sugerentes matices para justificar, además de su interés intrínseco, el derivado de su consideración como factor de vinculación entre los espacios universitarios y su entorno:

De notable interés parece la interpretación del parámetro flexibilidad: esta, de facto, se entiende no sólo y no tanto como posibilidad de transformación interna de los espacios para adecuarse a nuevas funciones, sino más bien como exigencia de los espacios formalmente cualificados en sentido autónomo, bien por características propias bien por relaciones formales con otros espacios, con el sistema de enlace, con el paisaje (De Carlo, 1968: 38).

A estas reflexiones se sumaban hace poco algunas investigadoras internacionales: «Ciertamente, el concepto de flexibilidad ha venido tomando importancia como herramienta de diseño, debido a la naturaleza dinámica y cambiante de los procesos de aprendizaje y enseñanza, los cuales demandan modelos espaciales capaces de adaptarse a requerimientos específicos» (Peñazola y Curvelo, 20II: II6). Por ende, la reiterada flexibilidad, como virtud aliada de la planificación, ha de descender hasta las escalas menores, donde deben generarse lugares de encuentro para la comunidad de aprendizaje: espacios polifacéticos, convertibles y abiertos. En I974, Unzurunzaga aportaba la siguiente argumentación, que sigue gozando hoy de vigencia: «La importancia de la flexibilidad, en contra de la rigidez que ha caracterizado a las escuelas tradicionales. Flexibilidad para conseguir una apertura, tanto humana como arquitectónica» (Unzurrunzaga, 1974: 35). Trasladando esta cualidad al plano académico, se ha constatado que una programación flexible y un espacio flexible expanden las iniciativas de los alumnos, despertándose un creciente interés por el aula inteligente (Segovia y Beltrán, 1998), de la cual se efectuó una posterior revisión (Segovia et al., 2003).

Planificar en clave flexible incide igualmente en la disposición del mobiliario y sus implicaciones perceptivas en el alumno. Resulta esencial que el tipo de muebles permita su cómoda y rápida redistribución dentro del ámbito áulico, según se vayan a impartir diferentes modalidades de Enseñanza/Aprendizaje. En general, los experimentos llevados a cabo por Sommer (Sommer, 1974) demostraron que los estudiantes prefieren inconscientemente las aulas con mobiliario flexible y en herradura, ya que se sienten más proclives a participar. En sentido opuesto, la rigidez propia de las aulas convencionales, con mesas y sillas fijadas al suelo, fomentan la insatisfacción y la pasividad.

Por regresar al tema-marco del presente epígrafe, debe apuntarse que la planificación se erige en garantía de unidad conceptual, traducible asimismo a la unidad ambiental. Las bondades de la elaboración de planes orientados a conferir orden y coherencia en los recintos docentes también han sido argumentadas por brillantes historiadores, como Bonet Correa: «Esencial en un campus es la planificación unitaria de su conjunto. Su disposición, con sus edificios jerarquizados, responde siempre a criterios funcionales muy estrictos a la vez que a razones de orden representativo» (Bonet, I995: 57). Así pues, planificar es una disciplina operativa que debe ajustarse a pautas funcionales, pero que también ha de observar unos ciertos protocolos de actuación, para poder optimizar sus aportaciones. Se deben concatenar una serie 
de acciones previas, como la fundamentación teórica y el análisis de la situación concreta. Así lo expresaba el recordado Castrejón:

La planificación para el cambio en una Universidad debe surgir de un proceso de reflexión y estudio sobre la misma, en el cual se le considere como un todo, puesto que será necesario que abarque los distintos aspectos de la Institución, como son el administrativo, financiero, académico, político y social (Castrejón, I990: I73).

Toda planificación debe seguir una dinámica de acción previamente definida. Es imprescindible efectuar un minucioso proceso de observación de todos los condicionantes, desarrollando una densa cavilación teórica; durante la fase proyectual, es aconsejable alcanzar un consenso entre los colectivos afectados; por último, debe acometerse una estrategia de comunicación para difundir adecuadamente las características del plan ideado. A partir de ese momento, podría procederse a la ejecución real de lo planificado, recomendando su estructuración en sucesivas etapas y contrastándose con los pertinentes sistemas de evaluación. Y, como último consejo, la planificación debiera revisarse con una determinada periodicidad y difundirse documentalmente. Es preciso recordar las valiosas aportaciones del arquitecto norteamericano Richard Dober:

Las claves de una buena planificación (universitaria) son bien conocidas; un bloque de información suficiente para emprender la tarea y una participación tan amplia como sea posible en el proceso [...] la esencial conjunción de esfuerzos necesarios para alcanzar y apoyar un consenso sobre lo que el futuro debería ser (Dober, 1996: 45).

En suma, la tarea planificadora es el más sólido aval para que la Universidad crezca con armonía espacio-temporal, alcanzando siempre un equilibrio entre el cambio y la continuidad. Debe efectuarse observando la premisa de que la concepción de un recinto universitario no es tanto la de un objeto urbanístico-arquitectónico, sino la de todo un proceso. Un proceso cambiante en el tiempo y en el espacio, como cambiante es el colectivo humano al que va dirigido, cuya motivación para con el aprendizaje se verá beneficiado por todo ello.

\section{La reivindicación de la escala humana en la universidad}

Una vez establecidos los argumentos básicos que avalan la planificación como actitud imprescindible en la educación superior, seguidamente procede analizar uno de sus objetivos prioritarios, cual es introducir ámbitos de escala humana. Con ello, se estarán sentando las bases para potenciar la cristalización de verdaderas comunidades de aprendizaje, factor decisivo en la motivación del estudiante y en el incremento de su sentimiento de pertenencia relativo a esta trascendental actividad en su vida. Para que, previa una insustituible labor planificadora, un recinto universitario genere el esperable ambiente de relación interpersonal y acogimiento, es quizá hoy más prioritario que nunca (por la creciente amenaza de la virtualidad), que incorpore espacios dotados de escala humana.

Como premisa, cabe recordar que el disfrute de la arquitectura exige su experiencia directa. Rasmussen señalaba: «Comprender la arquitectura no es, por lo tanto, lo 
LA OPTIMIZACIÓN DEL APRENDIZAJE EN LA UNIVERSIDAD A TRAVÉS DE SU ARQUITECTURA: PLANIFICACIÓN, ESCALA HUMANA Y COMUNIDAD VIVENCIAL PABLO CAMPOS CALVO-SOTELO

mismo que ser capaz de determinar el estilo de un edificio por ciertos rasgos exteriores. Esto no basta para ver la arquitectura, debe uno experimentarla» (Rasmussen, 1974: 37). Con frecuencia, los complejos universitarios tienen una gran extensión, como resultante de sus diferentes condicionantes. En tales situaciones, debe idearse una estrategia de ideación que combine la extensión global con su atomización en ámbitos reducidos, donde se fomente la cercanía. Esta característica, aparentemente relacionada solo con la dimensión física, está vinculada a una cualidad funcional: que se propicie la colectividad, como uno de los fundamentos del aprendizaje. La escala intimista despierta tanto el encuentro como la relación humana. Las personas involucradas en la trascendente actividad de la formación integral deben valerse de su engarce con otros individuos, pero también del diálogo visual y afectivo con diversos objetos (de entre los que destacan los de perfil urbanístico-arquitectónico): «Como la araña con su tela, cada individuo teje relaciones entre sí mismo y determinadas propiedades de los objetos; los numerosos hilos se entretejen y finalmente forman la base de la propia existencia del individuo» (Norberg-Schulz, I973: 7).

A partir de estas reflexiones básicas, procede avanzar en un aspecto que puede contribuir a que en el recinto se genere la deseable dimensión humana: caminar. La peatonalidad es una característica decisiva para que cristalice dicha dimensión. Caminar es el mejor procedimiento para gozar de un conjunto universitario. En oposición al frenético ir y venir cotidiano, andar pausadamente ayuda a conocer el lugar. El profesor López Silvestre apunta: «Pocas cosas nos siguen alejando más de la aplastante dinámica del consumismo urbano que un simple paseo sin destino claro» (López Silvestre, 2013: I5). Andar, como medio de desplazamiento, estimula además la apetencia por el aprendizaje, al existir una conexión entre el movimiento corporal y el incremento de la actividad mental. Algunos autores han indagado en el ejercicio físico como enriquecedor de la capacidad de aprender, ya que se suministra oxígeno al cerebro (Baumann y Boutellier, 20I2). Es hermoso deambular por un espacio universitario, detenerse y disfrutar del itinerario pausado. El filósofo decimonónico alemán Karl Gottlob Schelle, en su celebrado texto El Arte de pasear (I802), ensalzaba el paseo, ya que servía para: "Aunar actividad física y psíquica, elevar una actividad meramente mecánica -andar-al rango de una espiritual» (Schelle, 2013: 34).

Pero caminar incrementa también las relaciones sociales. Schelle recomendaba hacerlo para disfrutar, no únicamente de la Naturaleza, sino también de las personas. Más adelante, aquel sugerente ensayo cargado de poética invitaba a que: «Durante el paseo no se debe tensar la atención de la mente; ha de ser más bien un juego agradable antes que algo serio. Ha de vagar sobre los objetos con ligereza: antes responder a las solicitaciones de objetos de cualquier tipo que dejarse constreñir por la mente» (Schelle, 2013: 34).

Dentro de un complejo universitario, resulta recomendable desplazarse caminando, guiados hacia la meta funcional en cada momento. Pero, además, puede disfrutarse del navegar a la deriva, sin objetivo específico, dejándose llevar por la secuencia pausada de espacios; en suma, perderse, como recomendaba Walter Benjamin para recrearse en una zona urbana: "Y perderse en ella, como nos perdemos en un bosque, es cosa que hay que aprender» (Benjamin, I971: 76). El ya citado profesor López Silvestre añade otra recomendación: «En el momento en que vemos las cosas en relación a un fin o subordinadas al mismo, dejamos de verlas de otros modos. Pues bien, es ahí donde entra en escena el paseo relajado, que a causa de su naturaleza 
lúdica nos las muestra de modos variados» (López Silvestre, 2013: 17). La cita precedente justifica introducir el concepto de diversión. Apelando a su significado y origen etimológico, divertere remite a aquello que implica «llevar por varios lados» o también «dar giro en dirección opuesta», $\mathrm{y}$ "cambiar, dar la vuelta». Como derivada de ello, puede darse sentido a la diversión espacial, en tanto que inserción en la Universidad de lugares donde el alumno pueda entretenerse en actividades que rompan su rutina, abriendo su mente y su cuerpo a experiencias que le conduzcan hacia varios lados.

Los escenarios construidos de la Universidad se hallan salpicados de innumerables elementos que aportan valor. Entre ellos, las formas de la Naturaleza, las obras de arte y -esencialmente- las piezas arquitectónicas emergen en el paisaje general como hitos que propician el diálogo visual con el caminante. Este factor dialógico alimenta la verdadera experiencia vivencial, que se vale del paseo como mejor procedimiento. Y sin olvidar a las personas, que el mencionado Schelle asumía como ingredientes que aportan felicidad al caminar: «Naturaleza y género humano, la primera en sus escenarios más variopintos, el último en su forma más alegre, son a un tiempo el escenario y el objeto del paseo» (Schelle, 2013: 37). En otros términos: pasear enriquece la escala humana de la comunidad de aprendizaje, y sirve para afianzar los sentimientos de pertenencia del alumno; en suma, ayuda a sentir las vivencias con mayor intensidad. López Silvestre sugiere poéticamente que el paseo se torna: «Ciencia de la pierna y gastronomía del ojo» (López Silvestre, 2013: 7).

Planificar lugares de escala humana, cuya consecuencia es el refuerzo del sentimiento de pertenencia en los alumnos, implica tener en cuenta ciertas connotaciones perceptivo-psicológicas de las formas, como lo es el arquetipo de la envoltura espacial. Determinadas soluciones morfológicas generan en el usuario universitario sentimientos de albergue, protección y acompañamiento: una envoltura física que posee la capacidad de transmitir una paralela envoltura intelectual, e incluso afectiva. Como constatación histórica y actual del enriquecimiento que a una universidad aporta una adecuada inserción de ámbitos de escala humana, debe traerse a escena la estadounidense Universidad de Virginia. Desde hace años, la institución docente tomó la decisión de crear la Office of the Architect for the University. Pese a la pequeña dimensión de esta estructura de gestión, su cometido es de gran importancia, en relación a la preservación de la escala intimista. Compuesta únicamente por un arquitecto y una paisajista, su objetivo es velar por que cuantas obras y actuaciones se hagan en el emblemático recinto cuiden el espíritu del proyecto original de Thomas Jefferson, el Academical Village, tal y como fue ideado en i817. En España, la Universitat Politècnica de Catalunya ideó un ámbito nuevo, el Campus Nord, situado en el sector norte del recinto de la Diagonal. El núcleo principal se basa en una malla tridimensional regular, caracterizada por incorporar espacios libres cuya escala fue expresamente reducida, y que van alternándose con los volúmenes construidos en una armónica sucesión.

Los ejemplos mencionados, junto a muchos más que siembran el mapa internacional actual, testimonian que los espacios construidos de la Universidad deben adquirir para el estudiante el valor de lugares, esto es, añadiendo a la fría construcción una cálida componente de identificación personal. El universitario se afana en la búsqueda del conocimiento, y lo hace inmerso en un entorno edificado que debe contribuir tanto a dicha construcción intelectual como al cuidado emocional, generando formas 
LA OPTIMIZACIÓN DEL APRENDIZAJE EN LA UNIVERSIDAD A TRAVÉS DE SU ARQUITECTURA: PLANIFICACIÓN, ESCALA HUMANA Y COMUNIDAD VIVENCIAL PABLO CAMPOS CALVO-SOTELO

arquitectónicas que moldeen positivamente el comportamiento. Una emotividad que justifica cómo, a lo largo de la historia, la Universidad ha empleado morfologías arquitectónicas donde cristalizan metáforas humanas. Por ejemplo, el gesto cóncavo, resuelto mediante volúmenes de orientación centrípeta, protegiendo con paramentos laterales un interior delicado donde vive el ser humano. De ello dan fe la tipología claustral y su vasta herencia proyectual. La arquitectura, desempeñando un rol antropomórfico, evoca poéticamente el abrazo materno. Purini añade: «Tras las formas de la arquitectura didáctica aflora la referencia a la figura humana. El cuerpo está entendido no sólo como medida proporcional, sino como parámetro de todo el habitar, centro de una esfera de relaciones formales...» (Purini, I984: I83). Una vez que se ha introducido el antropomorfismo como cualidad adquirible por la arquitectura, es preciso insistir en el concepto de envoltura, desempeñable por los espacios universitarios, que ha llegado incluso a asignar al campus una propiedad metafórica como madre acogedora. Ello supone dar forma a una alegoría espacial del acogimiento, o adopción, a través de una ordenación que establezca impactos psicológicos que despierten empatías en el usuario. La disposición, volumen, forma y textura de las distintas piezas arquitectónicas en el recinto tendrán la misión de configurar realidades físicas que generen el deseable bienestar, asociable a la cristalización de una envoltura afectiva e intelectual. Uno de los factores de importancia es la acogibilidad, que engloba aspectos funcionales y estéticos. David Canter, entre otros, estudió el grado de satisfacción en el espacio docente teniendo en cuenta las respuestas a variables como las siguientes: estética (la valoración de los sujetos varía desde lo aburrido hasta lo impresionante); ornamentación (de alegre a triste); confort (de amistoso y confortable a hostil); organización (de eficiente y coordinado, a desordenado y caótico); potencia (de basto a leve o ligero); limpieza (de limpio a sucio); y espacio (de amplio a insuficiente) (Canter, 1978). Estos aspectos, a los que se deben sumar la disposición del mobiliario (en las aulas), la iluminación, el color o la ornamentación, poseen una considerable importancia desde el punto de vista del impacto afectivo de los espacios en el ser humano.

En suma, si en la planificación de un recinto universitario se toman las decisiones correctas, todas estas variables aumentarán la acogibilidad y mejorarán, por tanto, la impresión de que el alumno se funde en una auténtica envoltura afectivo-intelectual, que aportará la deseable escala humana a los espacios de la Universidad. Y contribuirá de este modo al afianzamiento de comunidades docentes, cuyos miembros interactúen bajo un compartido sentimiento de pertenencia.

\section{La cristalización de comunidades de aprendizaje e investigación: aproximación a unas posibles conclusiones}

El presente texto trata de justificar la importancia actual de tres cualidades, cuya puesta en acción podrá ayudar a optimizar la formación integral del estudiante (cual es la verdadera misión de las universidades): planificación, dimensión humana y sentido comunitario. Una tríada que, como se ha argumentado, se basa en la estrecha vinculación y concatenación de sus componentes.

La planificación de lugares de escala humana trae como consecuencia directa que se consoliden con mayor solidez comunidades de aprendizaje e investigación, dentro 
de las cuales se generen sentimientos de pertenencia a la Universidad, lo que redunda en la trascendental motivación para con el aprendizaje.

El presente artículo trata de demostrar que las conexiones conceptuales entre la referida tríada de principios del Campus Didáctico son una estrategia de alto interés para mejorar la calidad de la educación superior, en su comprensión como realidad integral afectada por la arquitectura. La verdadera formación integral de la persona solo puede alcanzarse en colectividad, puesto que es allí donde se estimula el contacto, verdadero sustento y nutriente de la relación interpersonal. Consolidar una comunidad vivencial de aprendizaje e investigación trae como consecuencia directa que prevalezca la dimensión humana en sus espacios, dentro de los cuales se genere el reiterado sentimiento de pertenencia. La planificación de un recinto debe llevarse a cabo dotando al conjunto de cuantos equipamientos y recursos sean necesarios para que se convierta en un ámbito donde estudiar, pero donde realizar también otras actividades: ocio, relación, deporte y -esencialmente- residencia.

Existe un beneficio inducido por el hecho de que los estudiantes aprendan en grupo, estando este formado asimismo por los otros perfiles que convergen en lo universitario: profesores, personal de administración y servicios y los ciudadanos. Este sencillo fundamento de partida ha sido subrayado por no pocos autores contemporáneos, como el arquitecto italoargentino Enrico Tedeschi, quien entendía ya hace décadas la Universidad incluso como un hábitat:

La Universidad se caracteriza por ser un lugar de convivencia organizada, poblado por personas diferentes en cuanto a edad, sexo, características sociales y culturales, que mantienen actividades distintas y que sin embargo se interaccionan. Por esto puede decirse que la universidad es un hábitat (Tedeschi, 1976: 22).

A ello cabe sumar una comprensión más vertical de estas comunidades docentes. Son numerosas las instituciones de educación superior que abren las puertas de sus recintos a colectivos ajenos, para que salgan beneficiados de la presencia universitaria. Como primer caso, la tercera edad, que puede integrarse dando cumplimiento al Aprendizaje a lo largo de toda la vida; pero asimismo se puede convocar a poblaciones infantiles. Es cada vez más habitual la incorporación de centros escolares, normalmente pertenecientes a la misma institución docente. La consecuencia es la convivencia de alumnos y profesores de los diferentes niveles de enseñanzas, superior y colegial. Surgen así atractivos paisajes humanos cargados de frescura y vitalidad, que dibujan un sugerente y vibrante perfil en la comunidad integral de aprendizaje.

La educación es un hecho colectivo, como se ha subrayado. Que el aprendizaje se facilite e incremente en colectividad es una circunstancia científicamente demostrable, a la que cabría superponer otra: la propia interacción constituye en sí misma un factor que aporta valores añadidos. En otras palabras: la comunidad enriquece la educación y es educativa en sí misma. En este sentido, cabe recordar algunos recintos ejemplares, donde se está persiguiendo con decisión el refuerzo de tales comunidades. Uno de ellos sería la Universidad Autónoma de Madrid, en su transformado recinto de Cantoblanco, que pasó de ser un complejo aislado y estacional a un ámbito cuya integralidad funcional está instando a un progresivo sentido comunitario. Y la californiana Universidad de Stanford que, trazada en I89i por Olmsted, se erigió en fiel representante del paradigma norteamericano en materia de fusión entre docencia, arquitectura y naturaleza; un campus que aún hoy se proyecta como paradigma de 
LA OPTIMIZACIÓN DEL APRENDIZAJE EN LA UNIVERSIDAD A TRAVÉS DE SU ARQUITECTURA: PLANIFICACIÓN, ESCALA HUMANA Y COMUNIDAD VIVENCIAL PABLO CAMPOS CALVO-SOTELO

vivencia, y cuyo crecimiento se halla estrechamente tutelado por la oficina de Land, Buildings and Real State ( $L B R E$ ), entidad interna comprometida con el realce de las virtudes históricas del complejo. Además de los ejemplos reseñados, está justificado hacer referencia a una plausible iniciativa surgida en universidades norteamericanas, las denominadas Learning communities. Estas estructuras internas, vinculadas a la idea de tutoría personal, llevan años alcanzando un notable éxito, en su tarea de ayudar a los estudiantes de cara a su inmersión académica y social. El profesor Knight ensalzaba las Learning communities así: «Están diseñadas para ayudar a que los estudiantes superen sentimientos de aislamiento que son frecuentes en grandes campus, y promover un sentimiento de identidad de grupo» (Knight, 2003: 6).

Comunidad de aprendizaje, sí. Y de investigación, también. Desde la institución fundada a inicios del xIx por Humboldt en Berlín, la búsqueda del saber mediante la investigación conjunta entre profesores y alumnos forma parte indisoluble de la Universidad. La génesis de nuevos conocimientos construye el progreso social. En tan importante misión, la labor coordinada entre los investigadores, susceptible de ser reforzada mediante el contacto presencial, contribuye a optimizar la propia actividad. Trabajar en comunidad trasciende a las disciplinas específicas de cada proyecto, al existir un denominador común derivado de la propia naturaleza intrínseca de la labor investigadora. No cabe duda de que los recintos universitarios, en virtud de su naturaleza física y del hecho de que acogen agrupaciones de Facultades o Escuelas, deben satisfacer no solamente las funciones estrictamente docentes e investigadoras, sino que -precisamente como cualidad derivada de su materialidad- han de fomentar el encuentro social. El profesor Ricard Pié, de la Universidad de Barcelona, lo subrayaba de este modo: «El campus puede ser el lugar físico donde se realiza la actividad universitaria, pero también un espacio de relación que genere conocimiento y especificidad» (Pié, 2004: I7).

Para que, previa su adecuada planificación, cristalice idóneamente una verdadera comunidad de aprendizaje e investigación (contribuyendo así a la conformación de un Campus Did́áctico), es preciso que se dote de integralidad funcional. Ello se traduce en la presencia de cuantos elementos sean precisos para generar entornos vivencialmente plenos, multifuncionales, que pongan a disposición del universitario cuanto necesite para su pleno desarrollo como ser social. Introducir la suficiente cantidad y variedad de recursos es el mejor instrumento para que se materialicen ambientes estimulantes, divertidos (en la acepción descrita), de dimensión humana, y alejados de la poco deseable estacionalidad. Como señalaba la arquitecta madrileña Maryan Álvarez-Builla:

La Universidad de las próximas décadas necesita, desde luego, contar con una cierta dotación propia de equipamientos, de manera que se pueda configurar un marco de vida suficiente para hacer atractiva la permanencia más allá de las actividades lectivas (Álvarez-Builla, I995: 242).

Los recintos universitarios han de valerse de dichos equipamientos generales que, integrados armónicamente, trasciendan al mero perfil docente e investigador: residenciales, lúdicos, deportivos, asistenciales, culturales, etc. Es decir, que se erijan en áreas de vitalidad integral, donde el estudiante se desarrolle como ciudadano, y dentro de las cuales pueda disfrutar del tiempo no dedicado a las tareas tradicionales. Si hay una función primordial en el listado anterior, esa es la residencia. Las estructuras 
habitacionales contribuyen muy eficazmente a que se incrementen los beneficios de la vida colectiva, el sentimiento de pertenencia y la motivación para la formación. Diversos autores han incidido en la trascendencia del babitar dentro del recinto docente, como factor clave para alcanzar una plena identificación de todos los usuarios con su institución. Tony Birks apuntaba: «Sin residencia, para personal universitario de toda índole, así como para estudiantes, no puede haber Universidad» (Birks, 1972: 25). La integralidad funcional es el más sólido aval para que se instituyan y afiancen estas comunidades de aprendizaje e investigación, traducibles a la consolidación de grupos humanos donde se propicie la verdadera comunicación, esto es, el intercambio de información mediante el diálogo, acompañado de una cierta carga emotiva. En función de la trascendencia que todo ello tiene de cara a optimizar el proceso de formación del futuro ciudadano, es esencial contar con un ámbito urbanístico-arquitectónico ideado a tal fin, de modo que se alcance un perfil vivencial pleno en el recinto: vivir, frente a un mero estar.

A modo de conclusión, es preciso insistir en que el escenario universitario actual exige un esfuerzo general para lograr optimizar su misión esencial: la formación integral de la persona. A tal fin, y dentro del marco teórico-práctico del Campus Didáctico, tres de sus principios pueden ensamblarse en la construcción de tal meta. Si se realiza una acción planificadora coherente y ambiciosa, podrán introducirse paulatinamente lugares urbanístico-arquitectónicos de escala humana en los recintos docentes, cuya consecuencia será el refuerzo de la motivación del alumnado, a través de la cristalización de verdaderas comunidades de aprendizaje e investigación.

La misión de la Universidad debe cumplirse también a través de su arquitectura.

\section{Bibliografía}

Abril, E. (20Io) Presentación. Locus Sapientiae. En vv. AA. La Universidad de Valladolid en sus edificios. Valladolid: Consejo Social Universidad de Valladolid.

Álvarez-Builla, M. (I995) El nuevo proyecto de alojamiento universitario en España. En vv. AA. La Ciudad del Saber. Ciudad, Universidad y Utopía. I293-1993 (pp. 239-254). Madrid: COAM.

Baumann, C. y Boutellier, R. (20I2) Physical Activity-The Basis of Learning and Creativity, En The Future of Education, Conference paper (pp. I-6). Florence, 201 .

Benjamin, W. (197I) Immagini di cittá. Turín: Einaudi.

BIRKs, T. (1972) Building the New Universities. London: David\&Charles.

Bonet Correa, A. (i995) De la Ciudad del Saber a la isla universitaria. En vv. AA. La Ciudad del Saber. Ciudad, Universidad y Utopía. I293-1993 (pp. 49-62). Madrid: COAM.

Campos, P. (2005) The University of Salamanca's new campus. PEB-Exchange. Programme on Educational Building, OECD Publishing. http://dx.doi.org/I0.1787/544636403567, 2005 (I2), 7-9.

Campos, P. (2009) La Educación, un hecho espacial. El Campus Didáctico como Arquitectura para el Espacio Europeo de Educación Superior. La Cuestión Universitaria. Madrid: Cátedra unesco-Universidad Politécnica de Madrid, 2009 (5), 99-I2I.

Campos, P. (20IOa) Io Principles of an innovative model for the 2ist century University: the Educational Campus. Aula, Revista de Pedagogía de la Universidad de Salamanca, núm. I6, 187-200.

Campos, P. (2orob) España-campus de Excelencia Internacional. Madrid: Ministerio de Educación. 
LA OPTIMIZACIÓN DEL APRENDIZAJE EN LA UNIVERSIDAD A TRAVÉS DE SU ARQUITECTURA: PLANIFICACIÓN, ESCALA HUMANA Y COMUNIDAD VIVENCIAL PABLO CAMPOS CALVO-SOTELO

Campos, P. (2012) Composición Arquitectónica: Fundamentos teóricos y aplicaciones en los espacios para la educación. Madrid: cEU Ediciones.

Campos, P. (20I4) From typological analysis to planning: modern strategies for University spatial quality. CIAN-Revista de Historia de las Universidades, I7 (I), 31-58.

Campos, P. (2015) Niveles espaciales y dimensión fenomenológica en los ámbitos universitarios. Arquitectonics. Mind, LandESociety. Arquitectura, Fenomenología y Dialogía Social, núm. 27.

Campos, P. (20I7a) Reflexiones en torno a la arquitectura del aula universitaria: dimensión didáctica, fenomenología y disolución de los límites espacio-temporales en la optimización de comunidades de aprendizaje. RELAPAE, núm. 6, mayo.

Campos, P. (2017b) El paradigma del «Campus Didáctico»: revisión conceptual y proyección en los espacios físicos de la Universidad. (Tesis Doctoral inédita). Departamento de Teoría e Historia de la Educación. Universidad de Salamanca.

CANO, J. (1993) El entono de los edificios universitarios. Urbanismo-COAM, núm. 2I, 3I-36.

CANTER, D. (1978) Interacción ambiental. Alcalá de Henares: Instituto Nacional Administración Pública.

Castrejón, J. (1990) El concepto de Universidad. México: Editorial Trillas.

De Carlo, G. (1968) Planificazione e Disegno delle Università. Roma: Edizione Universitarie Italiane.

Dober, R. (1996) Campus planning. Ann Arbor, mI: Society for College and University Planning-SCUP.

Gaines, T. (1991) The Campus as a Work of Art. Westport, ст: Praeger Publishers.

Knight, W. (2003) Learning communities and first-year programs. Planning for Higher Education, vol. 3I (4), 5-I2.

Kong, S. Y.; YAacob, N. M. y Ariffin, A. R. M. (20I5) Physical environment as a 3-D textbook: design and development of a prototype. Asia Pacific Journal of Education, 35 (2), 24I-258.

LAeng, M. (1977) Esquemas de pedagogía. Barcelona: Herder.

López Silvestre, F. (ed.) (2013) El mundo a tres kilómetros por hora. En El Arte de pasear. Madrid: Díaz \& Pons Editores.

Norberg-Schulz, C. (1973) Arquitectura occidental. La arquitectura como historia de las formas significativas. Barcelona: Gustavo Gili.

Peñaloza, A. y Curvelo, F. (20iI) La experiencia del espacio académico flexible BK-City, Universidad Técnica de Delft, laboratorio espacial de una Facultad de arquitectura. Revista dearq, 9. Bogotá: Universidad de los Andes, II4-I3I.

PIÉ, R. (2004) La Universitat en el territorio: reflexió histórica i consideracions sobre el cas català. Coneixement i Societat. Revista d'Universitats, Recerca i Societat de la Informació. I. ${ }^{\circ}$ quadrimestre. Generalitat de Catalunya, I6-43.

Purini, F. (1984) La Arquitectura didáctica. Colegio Oficial de Aparejadores y Arquitectos técnicos de Murcia. Versión original: Casa del libro Editrice, I980.

Rasmussen, S. E. (1974) Experiencia de la Arquitectura. Barcelona: Editorial Labor. Versión original: Experiencing Architecture. Cambridge, MA: MIT Press, 1959.

Rebecchini, M. (198i) Progettare L'Università. Roma: Edizioni Kappa.

Romañá, T. (2004) Arquitectura y Educación: perspectivas y dimensiones. Revista Española de Pedagogía, LXII, 228, 199-220.

Schelle, K. G. (2013) El Arte de pasear. En F. López Silvestre El mundo a tres kilómetros por hora. Madrid: Díaz \& Pons Editores.

Segovia, F. y Beltrán, J. (1998) El Aula Inteligente. Nuevo horizonte educativo. Madrid: Espasa Calpe.

Segovia, F. et al. (2003) El Aula Inteligente. Nuevas perspectivas. Madrid: Espasa Calpe.

Sommer, R. (1974) Espacio y comportamiento individual. Madrid: Instituto de Estudios de Administración Local. 
Tedeschi, E. (1976) La Universidad como hábitat. Summa, núm. Io4, 22-38.

Unzurrunzuaga, M. T. (1974) Consecuencias arquitectónicas de las nuevas tendencias pedagógicas. Revista de Educación, 223-224, 34-53.

Waisman, M. (1995) La Universidad en la Ciudad: la recuperación de edificios históricos. En vv. AA. La Ciudad del Saber. Ciudad, Universidad y Utopía. I293-1993 (pp. 63-70). Madrid: COAM. 